\title{
Model of metastable EeV dark matter
}

\author{
Emilian Dudas, ${ }^{1, *}$ Lucien Heurtier®, ${ }^{2, \dagger}$ Yann Mambrini, ${ }^{3, *}$ Keith A. Olive, ${ }^{4,8}$ and Mathias Pierre $\circledast^{5,6, \|}$ \\ ${ }^{1}$ Centre de Physique Théorique, École Polytechnique, CNRS and IP Paris, 91128 Palaiseau Cedex, France \\ ${ }^{2}$ Department of Physics, University of Arizona, Tucson, Arizona 85721, USA \\ ${ }^{3}$ Université Paris-Saclay, CNRS/IN2P3, IJCLab, 91405 Orsay, France \\ ${ }^{4}$ William I. Fine Theoretical Physics Institute, School of Physics and Astronomy, \\ University of Minnesota, Minneapolis, Minnesota 55455, USA \\ ${ }^{5}$ Instituto de Física Teórica (IFT) UAM-CSIC, Campus de Cantoblanco, 28049 Madrid, Spain \\ ${ }^{6}$ Departamento de Física Teórica, Universidad Autónoma de Madrid (UAM), \\ Campus de Cantoblanco, 28049 Madrid, Spain
}

(Received 16 March 2020; accepted 11 June 2020; published 22 June 2020)

\begin{abstract}
We propose a model where a long-lived pseudoscalar EeV particle can be produced with sufficient abundance so as to account for the cold dark matter density, despite having a Planck mass suppressed coupling to the thermal bath. Connecting this state to a hidden sterile neutrino sector through derivative couplings, induced by higher dimensional operators, allows one to account for light neutrino masses while having a lifetime that can be much larger than the age of the Universe. Moreover, the same derivative coupling accounts for the production of dark matter in the very first instant of the reheating. Given the sensitivity of the IceCube and ANITA collaborations, we study the possible signatures of such a model in the form of ultrahigh-energy cosmic rays in the neutrino sector, and show that such signals could be detected in the near future.
\end{abstract}

DOI: 10.1103/PhysRevD.101.115029

\section{INTRODUCTION}

Despite many efforts, the presence of dark matter (DM) in the Universe [1] has not yet been confirmed by any direct [2-4] or indirect [5-9] detection signal. Recent limits severely constrain typical WIMP scenarios such as the Higgs-portal [10,11], Z-portal [12], or even the $Z^{\prime}$-portal [13]. More complex extensions such as the minimal supersymmetric standard model [14-16] also have a large part of their parameter space excluded [17-19] from LHC searches [20]. Direct, indirect and accelerator searches place additional constraints on these models (for a review on WIMP searches and models, see [21]). As a consequence, it is important to look for alternative scenarios, including those with ultraweak couplings such as gravitinos $[16,22]$ or Feebly Interacting Massive Particles (FIMP's) [23] (see [24] for a review), highly-decoupled dark sectors [25], or the possibility that DM production occurred in the

\footnotetext{
*emilian.dudas@polytechnique.edu

†heurtier@email.arizona.edu

‡yann.mambrini@th.u-psud.fr

olive@umn.edu

"mathias.pierre@uam.es
}

Published by the American Physical Society under the terms of the Creative Commons Attribution 4.0 International license. Further distribution of this work must maintain attribution to the author(s) and the published article's title, journal citation, and DOI. Funded by SCOAP. very early stages of reheating after inflation as in $\mathrm{SO}(10)$ grand unification [26,27], anomaly free U(1)' models [28], spin-2 portals [29], high scale supersymmetry [30-35] or even moduli portals [36]. In all of these models, it has been shown that the effects of noninstantaneous reheating $[31,37,38]$ and the noninstantaneous thermalization of reheating products [39] on the production of DM particles are non-negligible.

On the other hand, the absolute stability of DM is usually justified by imposing a symmetry. Discrete symmetries are the most popular (R-parity in supersymmetry [40], a $Z_{2}$ symmetry in $S O(10)$ unification [27,41], a $Z_{2}$ symmetry in Higgs [10,11] or Z-portal [12] models) and can arise from broken gauge symmetries which are exact at the Planck scale. This is not the case for continuous global symmetries which are generically violated at the Planck scale [42-45]. In this case, the decay of DM is rendered possible through Planck-suppressed operators, as argued in [46].

Due to its very specific signature (monochromatic final states for a 2-body decay), a metastable candidate is regularly evoked when specific detection signals are claimed. For example, a positron excess [47], photon lines [48], high energy neutrinos in IceCube $[49,50]$ or ultrahigh energy neutrinos in ANITA [51,52]. However, in each case, the interpretation of a signal as a dark matter detection has to deal with a severe issue: justifying a long lifetime (and thus extremely tiny couplings) while at the same time finding a production mechanism able to produce the dark 
matter in a sufficiently large amounts to account for the PLANCK determined density of dark matter [1] (implying a coupling which is not so tiny).

At first sight, it would seem that Planck-suppressed couplings of DM particles to Standard Model (SM) states could be sufficient for explaining why dark matter may be long lived on cosmological time scales. For example, one may naively expect the decay width of dark matter to be of order $\Gamma \simeq \frac{m^{3}}{M_{p}^{2}}$ where $m$ denotes the DM mass. ${ }^{1}$ However, given the current limit from indirect gamma [53] positron [54] or neutrino [55] detection ( $\tau=\Gamma^{-1} \gtrsim 10^{29}$ seconds) one would require $m \lesssim 10 \mathrm{keV}$ which reaches the limit from Lyman- $\alpha$ or structure formation constraints [56]. It is, moreover, not an easy task to produce the requisite abundance of DM particles with such feeble couplings. Even the FIMP scenario necessitates couplings of the order of $10^{-11}$ [23], i.e., much larger than $\frac{m^{2}}{M_{P}^{2}}$.

A potentially more natural way to couple DM to the Standard Model (SM) bath with Planck suppressed couplings is through the neutrino sector, for which there are already strong mass constraints $\sum m_{\nu} \lesssim 0.15 \mathrm{eV}$ [57]. Indeed, several constructions invoke a new massive scalar [58] to justify the neutrino mass through a dynamical process similar to the Higgs mechanism applied in the right-handed neutrino sector.

In this work, we show that by combining the violation of global continuous symmetries at the Planck scale, while coupling DM to the neutrino sector, one can generate a large DM lifetime

$$
\tau \propto\left(\frac{M_{P}}{m_{\nu}}\right)^{2} \frac{1}{m} \simeq 10^{33} \mathrm{~s}\left(\frac{0.05 \mathrm{eV}}{m_{\nu}}\right)^{2}\left(\frac{1 \mathrm{GeV}}{m}\right),
$$

in compliance with the actual experimental constraints for $\sim 1 \mathrm{PeV}$ dark matter masses. ${ }^{2}$ The paper is organized as follows. In Sec. II we present our model and we compute the DM lifetime and relic abundance in Sec. III. We propose experimental signatures in Sec. IV. In Sec. V, we propose a top-down model which incorporates all of the needed components for our EeV DM candidate and its coupling to the observational sector. Our conclusions are given in Sec. VI. Appendix A contains additional details on the computation of the decay rates and Appendix B gives more detail on the UV microscopic model containing additional particles and interactions which generate, in the IR, the effective model with appropriate mass scales and couplings that we analyze in the bulk of the paper.

\footnotetext{
${ }^{1}$ We will use the reduced Planck mass throughout the paper, $M_{P}=\frac{1}{\sqrt{8 \pi G_{N}}}=2.4 \times 10^{18} \mathrm{GeV}$.

${ }^{2}$ Note that the lifetime of the Universe is $\sim 4 \times 10^{17}$ seconds, corresponding to $\sim 6.5 \times 10^{41} \mathrm{GeV}^{-1}$ whereas limits from indirect detection reach $\tau \gtrsim 10^{29}$ seconds $\left(\sim 10^{53} \mathrm{GeV}^{-1}\right)$.
}

\section{THE MODEL}

\section{A. Motivations}

We begin with some motivation for the general and more detailed models we present below. The models we are proposing rely on a derivative coupling of a DM candidate, $a$, to matter. Indeed, axionic couplings of the type $\frac{\alpha}{M_{P}} \partial_{\mu} a$ appears in several ultraviolet constructions. For instance, in models with string or higher-dimensional inspired moduli fields $T=t+i a$ (see [36] for a more detailed study), they can couple to a sterile sector through the kinetic term as

$$
\mathcal{L} \supset \frac{i}{2}\left[\bar{\nu}_{s} \gamma^{\mu} \mathcal{Z}_{s} \partial_{\mu} \nu_{s}-\left(\partial_{\mu} \bar{\nu}_{s}\right) \gamma^{\mu} \mathcal{Z}_{s}^{*} \nu_{s}\right]
$$

with $\mathcal{Z}_{s}=1+\frac{\beta_{s}}{M_{P}} t+i \frac{\alpha_{s}}{M_{P}} \gamma_{5} a$ and $\alpha_{s}, \beta_{s}$ real for simplicity. After an integration by parts, the Lagrangian will contain terms

$$
\mathcal{L} \supset \frac{\alpha_{s}}{2 M_{P}}\left(\partial_{\mu} a\right) \bar{\nu}_{s} \gamma^{\mu} \gamma^{5} \nu_{s}
$$

which are of the form we consider below.

We can also find such couplings in the Majoron model. Consider a Lagrangian of the type

$$
\mathcal{L}_{\phi}=\phi \nu_{s} \nu_{s}+\text { H.c. }
$$

written using a two-component notation and where $\phi=$ $\chi e^{\frac{i a}{M_{P}}}$ is the Majoron. After a redefinition of phases, $\nu_{s} \rightarrow e^{-\frac{i a}{2 M_{P}}} \nu_{s}$, the kinetic term, $-i \bar{\nu}_{s} \bar{\sigma}^{\mu} \partial_{\mu} \nu_{s}$, produces a coupling of the type given in Eq. (3) [50,59].

Even in string constructions, where we can define the moduli superfield in term of the Grassmannian variables $\theta$ and $\bar{\theta}$ by $T+\bar{T}=2 t+2 \theta \sigma^{\mu} \bar{\theta} \partial_{\mu} a$, we can show that a term $\frac{1}{\left\langle t^{2}\right\rangle M_{P}} \partial_{\mu} a \bar{\nu}_{s} \sigma^{\mu} \nu_{s}$ appears once expanding the Kähler metric as function of matter fields. In this case, $\alpha_{s}$ can be identified as $\frac{1}{\left\langle t^{2}\right\rangle} \simeq 10^{-2}-10^{-3}$ in KKLT-like models [60]. As one can see, several ultraviolet constructions contains couplings of the type (3) which we use below.

\section{B. The Lagrangian}

Our goal in this section is to build a minimal model of metastable $\mathrm{EeV}$ dark matter. By minimal, we mean that we introduce the fewest number of new fields beyond those in the Standard Model with neutrino masses. We assume that DM is a pseudoscalar field. As alluded to above, the most economical and natural way to proceed is to couple the pseudo-scalar to a sterile neutrino $\left(\nu_{s}\right)$ and/or right-handed neutrino $\left(\nu_{R}\right)$ sector $^{3}$ as is the case for the pseudo-scalar part of the Majoron. We consider the following Lagrangian

\footnotetext{
${ }^{3}$ This coupling can be justified in models with large extra dimensions, where SM is localized on a brane, whereas gravity and SM singlets, in particular sterile neutrinos, propagates into a bulk internal space [61] and couple to an axion localized on a distant brane.
} 


$$
\mathcal{L}=\mathcal{L}_{\Phi}+\mathcal{L}_{s}+\mathcal{L}_{R}
$$

with

$$
\begin{gathered}
\mathcal{L}_{\Phi}=y_{f} \Phi \bar{f} f+\left(y_{\phi} \Phi \bar{\nu}_{s}^{c} \nu_{s}+\text { H.c. }\right) \\
\mathcal{L}_{s}=\frac{\alpha}{M_{P}} \partial_{\mu} a \bar{\nu}_{s} \gamma^{\mu} \gamma^{5} \nu_{s}-\left(y_{s} \tilde{H} \bar{L} \nu_{s}+\frac{1}{2} m_{s} \bar{\nu}_{s}^{c} \nu_{s}+\text { H.c. }\right)
\end{gathered}
$$

$\mathcal{L}_{R}=\frac{\alpha}{M_{P}} \partial_{\mu} a \bar{\nu}_{R} \gamma^{\mu} \gamma^{5} \nu_{R}-\left(y_{R} \tilde{H} \bar{L} \nu_{R}+\frac{1}{2} M_{R} \bar{\nu}_{R}^{c} \nu_{R}+\right.$ H.c. $)$.

In (7), we have included a Yukawa coupling, $y_{s}$ for the sterile neutrino to a $S U(2)_{L}$ Standard Model doublet, $L$ and the Higgs doublet, $H$, giving rise to a Dirac mass term. We also include a Majorana mass term for $\nu_{s}$. In (8), in addition to coupling the pseudo-scalar to $\nu_{R}$, we add the standard Dirac and Majorana mass terms needed for the seesaw mechanism [62].

As it will be important later when we discuss the production of dark matter during reheating, we also introduce an inflaton, $\Phi$, and couple it to both the sterile sector and the SM, where $f$ corresponds to a SM fermion. Finally, $\alpha$ is a coupling $\lesssim 1$ that represents the physics behind the Planck suppressed terms.

The Lagrangian terms in Eqs. (7) and (8) lead to the following neutrino mass matrix:

$$
\frac{1}{2}\left(\begin{array}{lll}
\bar{\nu}_{L} & \bar{\nu}_{s}^{c} & \bar{\nu}_{R}^{c}
\end{array}\right)\left(\begin{array}{ccc}
0 & m_{D}^{s} & m_{D}^{R} \\
m_{D}^{s} & m_{s} & 0 \\
m_{D}^{R} & 0 & M_{R}
\end{array}\right)\left(\begin{array}{c}
\nu_{L} \\
\nu_{s}^{c} \\
\nu_{R}^{c}
\end{array}\right)+\text { H.c. }
$$

where $m_{D}^{s}=y_{s} v_{h} / \sqrt{2}, m_{D}^{R}=y_{R} v_{h} / \sqrt{2}$ and $v_{h}=246 \mathrm{GeV}$ is the SM Higgs vacuum expectation value. We assumed flavor-diagonal couplings in the SM neutrino sector for simplicity and suppressed flavor indices. We also assume the following mass hierarchy (that will be justified in Sec. V)

$$
m_{s}<m_{D}^{R} \ll M_{R} .
$$

After diagonalization, we can define the 3 mass eigenstates $\nu_{1}, \nu_{2}, \nu_{3}$ by

$$
\begin{aligned}
& \nu_{1}=\cos \theta\left(\nu_{s}+\nu_{s}^{c}\right)+\sin \theta\left(\nu_{L}+\nu_{L}^{c}\right), \\
& \nu_{2}=\cos \theta\left(\nu_{L}+\nu_{L}^{c}\right)-\sin \theta\left(\nu_{s}+\nu_{s}^{c}\right), \\
& \nu_{3} \sim \nu_{R},
\end{aligned}
$$

with $^{4}$

\footnotetext{
${ }^{4}$ Using the approximation $m_{s} M_{R} \ll\left(m_{D}^{R}\right)^{2}$
}

$$
\tan 2 \theta=\frac{2 m_{D}^{s} M_{R}}{\left(m_{D}^{R}\right)^{2}+M_{R} m_{s}} \simeq \frac{2 m_{D}^{s}}{m_{1}+m_{2}},
$$

which implies that

$$
y_{s} \simeq \sqrt{2} \theta \frac{m_{1}+m_{2}}{v} \simeq \sqrt{2} \theta \frac{m_{2}}{v} \lesssim 2.9 \times 10^{-13} \theta
$$

and

$$
m_{1} \simeq m_{s}, \quad m_{2} \simeq \frac{\left(m_{D}^{R}\right)^{2}}{M_{R}}, \quad m_{3} \simeq M_{R},
$$

where the last inequality in (12) assumes a SM-like neutrino mass of $m_{2}=0.05 \mathrm{eV}$.

In the $\nu_{1}, \nu_{2}$ basis, we can rewrite the Lagrangian couplings of $a$ and $\Phi$ to the light neutrino sector as

$\mathcal{L}=\alpha \frac{\partial_{\mu} a}{M_{P}}\left(\bar{\nu}_{1} \gamma^{\mu} \gamma_{5} \nu_{1}-\theta\left(\bar{\nu}_{2} \gamma^{\mu} \gamma_{5} \nu_{1}+\overline{\nu_{1}} \gamma^{\mu} \gamma_{5} \nu_{2}\right)+\mathcal{O}\left(\theta^{2}\right)\right)$,

and

$$
\mathcal{L}=y_{\phi} \Phi\left(\bar{\nu}_{1} \nu_{1}-\theta\left(\bar{\nu}_{1} \nu_{2}+\bar{\nu}_{2} \nu_{1}\right)+\mathcal{O}\left(\theta^{2}\right)\right)
$$

As one can see, our framework is similar to a double seesaw mechanism, and the coupling of the dark matter to the standard model will be highly dependent on the mixing angle $\theta$. Even if couplings of the form in Eq. (7) may seem ad hoc, they can in fact be justified by high-scale motivated models, an example of which is given in Sec. V.

\section{THE CONSTRAINTS}

In this section, we consider several necessary constraints on the model. These include constraints on the lifetime from indirect detection searches, constraints on the DM abundance- that is we require a viable production mechanism, and cosmological constraints on the sterile sector from contributions to the effective number of neutrino degrees of freedom, $N_{\text {eff }}$.

\section{A. Lifetime constraints}

The first constraint we apply to the model is on the lifetime of the dark matter candidate $a$. To be a viable DM candidate, $a$ should at least live longer than the age of the Universe. However, as was shown in [50], when dealing with long-lived decays of particles to the neutrino sector, many body final state decays can dominate over two-body decays when a spin flip makes the amplitude proportional to the neutrino mass in the final state. This is reminiscent of three-body annihilation processes generated by internal brehmshtralung which dominate over two-body 
annihilation processes suppressed for light fermionic final states due to spin-momentum constraints.

In principle, there are two lifetime limits of importance. First, the DM lifetime (to any final state) must be longer than the age of the Universe $\left(\tau_{a}>4 \times 10^{17} \mathrm{~s}\right)$. Second, the lifetime must exceed $10^{29} \mathrm{~s}$ when there is an observable neutrino in the final state $[63,64]$. In our case (see Appendix A for details), the dominant decay channel is indeed the three-body final state decay $\Gamma_{a \rightarrow \nu_{1} \nu_{2} h / Z}$ and $\Gamma_{a \rightarrow \nu_{1} e W}$. All three of these modes have similar amplitudes. Note that we are interested in final states where a SM particle appears, especially an active neutrino, as that gives us the most stringent constraints from experiment. ${ }^{5}$ The $\nu_{1} \nu_{2} h$ final state is most important and we obtain (see Appendix A)

$$
\Gamma_{a \rightarrow \nu_{1} \nu_{2} h}=\frac{\alpha^{2} \theta^{2} m_{a}^{3}}{192 \pi^{3} v^{2} M_{P}^{2}}\left(m_{1}+m_{2}\right)^{2},
$$

implying that

$\tau_{a} \gtrsim 5.5 \times 10^{28} \mathrm{~s}\left(\frac{10^{-2}}{\alpha}\right)^{2}\left(\frac{10^{-5}}{\theta}\right)^{2}\left(\frac{10^{9} \mathrm{GeV}}{m_{a}}\right)^{3}$

for $m_{1} \ll m_{2} \lesssim 0.05 \mathrm{eV}$. Note first the rather amazing result that a pseudoscalar with mass $10^{9} \mathrm{GeV}$, has a lifetime which greatly exceeds the age of the Universe. This is due primarily to the Planck suppressed coupling and the neutrino mass (squared) in the decay rate. Note also that the lifetime of $a$ is determined by the mixing angle $\theta$ which we have normalized to $10^{-5}$ requiring a relatively small Yukawa coupling of order $10^{-18}$ from Eq. (12). The smallness of $y_{s}$ will be justified in Sec. V. In this way, we avoid taking $\alpha$ excessively small. ${ }^{6}$

Limits from [64] gives $\tau_{a \rightarrow \nu_{2} \nu_{2}} \gtrsim 5 \times 10^{28}$ seconds whereas [63] obtained $\tau_{a \rightarrow b \bar{b}} \gtrsim 10^{29}$ seconds. To be as conservative as possible, we will consider the upper limit $m_{2}=0.05 \mathrm{eV}$ for the neutrino mass and $\tau_{a} \gtrsim 10^{29}$ seconds throughout our work.

\section{B. Cosmological constraints}

Another important constraint comes from the relic abundance of the dark matter. Unless one heavily finetunes the coupling of the inflaton to $a$, the direct production of $a$ through (two-body) inflaton decay would greatly overproduce the density of $a$ whose annihilation rate would be extremely small. That is, we cannot rely on any kind of thermal freeze-out scenario. It is however possible to produce $a$ in sufficient quantities through the three-body

\footnotetext{
${ }^{5}$ Note that the dominant 2-body decay has $\nu_{1} \nu_{1}$ in the final state. But for $m_{2} \theta>10^{-5} m_{1}$, the three body partial width is always larger (see Eq. (A9) in Appendix A).

${ }^{6}$ Indeed, the way we wrote the Planck mass coupling $\frac{\alpha}{M_{P}}$ imposes $\alpha \lesssim 1$ to avoid large trans-Planckian BSM scales.
}

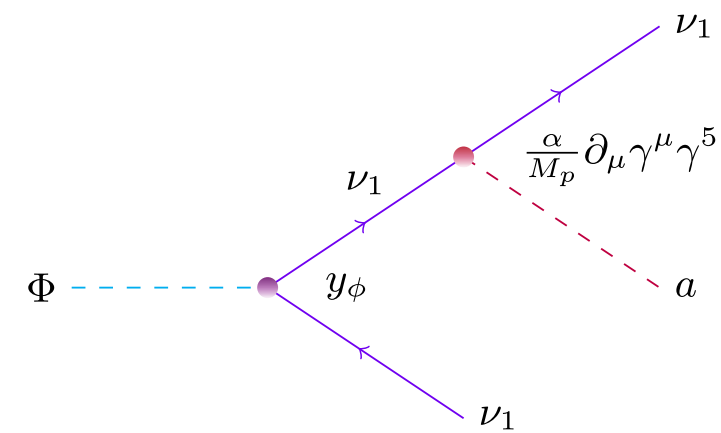

FIG. 1. Main inflaton decay channel contributing to the production of dark matter.

decay of the inflaton coupled only to SM fermions and the sterile sector as in Eq. (6). This allows for a decay channel $\phi \rightarrow a \nu_{1} \nu_{1}$ as shown in Fig. 1.

We assume here some rather generic features of the inflationary sector, and do not need to specify a particular model. We assume a coupling of the inflaton to the SM so that reheating is achieved (we assume instantaneous reheating and thermalization). If dominant, the decay rate is given by

$$
\Gamma_{\Phi \rightarrow \bar{f} f}=\frac{y_{f}^{2} N}{8 \pi} m_{\Phi}
$$

where $N$ is an effective number of final state fermionic degrees of freedom and is similar to the total number of degrees of freedom of the Standard Model. If dominant, this decay leads to a reheating temperature given by

$$
T_{\mathrm{RH}}=\left(\frac{5 N}{8 \pi^{4}}\right)^{1 / 4} y_{f} \sqrt{M_{P} m_{\Phi}} .
$$

In general, the relic abundance of dark matter produced in inflaton decay with a branching ratio $B_{R}$ can be expressed as [35]

$\Omega_{a} h^{2} \simeq 0.1\left(\frac{B_{R}}{9 \times 10^{-16}}\right)\left(\frac{3 \times 10^{13}}{m_{\Phi}}\right)\left(\frac{T_{\mathrm{RH}}}{10^{10}}\right)\left(\frac{m_{a}}{10^{9}}\right)$,

where all masses are expressed in $\mathrm{GeV}$. In our specific case, the partial width for producing the DM candidate $a$ is the three body decay width

$$
\Gamma_{\Phi \rightarrow a \bar{\nu}_{1} \nu_{1}}=\frac{\alpha^{2} y_{\phi}^{2}}{24 \pi^{3}}\left(\frac{m_{\Phi}}{M_{P}}\right)^{2} m_{\Phi},
$$

and the branching ratio for $\Phi \rightarrow \bar{\nu}_{1} \nu_{1} a$ is given by 


$$
\begin{aligned}
B_{R} & =\frac{\Gamma_{\Phi \rightarrow \bar{\nu}_{1} \nu_{1} a}}{\Gamma_{\Phi \rightarrow \bar{f} f}} \\
& \simeq \frac{5 \times 10^{-16}}{N}\left(\frac{\alpha}{10^{-2}}\right)^{2}\left(\frac{y_{\phi}}{y_{f}}\right)^{2}\left(\frac{m_{\Phi}}{3 \times 10^{13}}\right)^{2},
\end{aligned}
$$

where we have assumed that the total rate is dominated by the two-body decay to SM fermions, or equivalently that $N y_{f}^{2} \gg y_{\phi}^{2}$.

Implementing the expression for the branching ratio into Eq. (20) we obtain

$$
\begin{aligned}
\Omega_{a} h^{2} \simeq & 0.1 \times \frac{125}{N}\left(\frac{\alpha}{5 \times 10^{-2}}\right)^{2}\left(\frac{y_{\phi}}{y_{f}}\right)^{2}\left(\frac{m_{\Phi}}{3 \times 10^{13} \mathrm{GeV}}\right) \\
& \times\left(\frac{T_{\mathrm{RH}}}{10^{11} \mathrm{GeV}}\right)\left(\frac{m_{a}}{10^{9} \mathrm{GeV}}\right) .
\end{aligned}
$$

We note that the expression (23) does not depend on the parameter $\theta$, in contrast to the lifetime of $a$ (16). Indeed, the dominant decay channel of the inflaton to neutrinos involves only the lighter state, whereas mixing proportional to $\theta$ is compulsory for decays with $\nu_{2}$ in the final state. We also note that to produce the Planck determined abundance of EeV DM, we need $T_{\mathrm{RH}} \sim 10^{11} \mathrm{GeV}$ when the Yukawa couplings, $y_{\Phi}$ and $y_{f}$ are similar. $^{7}$

\section{Constraints on $N_{\text {eff }}$}

It is also important to consider the contribution of neutrino sector present in our model to the overall expansion rate of the universe. In principle, adding a new light degree of freedom, would increase the effective number of light neutrinos which is strongly constrained by the $\mathrm{CMB}$ and BBN. The current upper limit is [65]

$$
\Delta N_{\text {eff }}<0.17 \quad(95 \% \mathrm{CL}),
$$

where $\Delta N_{\text {eff }}=N_{\text {eff }}-3$. However, a completely sterile neutrino which would never equilibrate with the SM bath would only contribute a small fraction of a neutrino to $\Delta N_{\text {eff }}$ since its energy density gets greatly diluted compared to the energy density of SM neutrinos [66]. In cases where a light sterile neutrino (or right-handed $\nu_{R}$ ) mixes with the active left-handed neutrinos $\nu_{L}$, a non-negligible contribution to $N_{\text {eff }}$ may result.

Using Eq. (24), we can derive an upper limit on the mixing angle, $\theta$, by noting that interaction rates for $\nu_{1}$ are the same as those of active neutrinos, $\nu_{2}$, suppressed by $\theta^{2}$. Therefore, $\nu_{1}$ will decouple at a higher temperature, $T_{d 1}$, than that of $\nu_{2}, T_{d 2}$. Indeed we can appoximate

\footnotetext{
${ }^{7}$ It is worth mentioning that an alternative possibility would be to produce dark matter only through the graviton-portal but at the price of requiring a very large reheating temperature $\left(T_{\mathrm{RH}} \gtrsim 10^{14} \mathrm{GeV}\right)$ as was shown in [29]).
}

$T_{d 1} \theta^{2 / 3}=T_{d 2}=2 \mathrm{MeV}$. As a result, the ratio of the temperatures of $\nu_{1}$ and $\nu_{2}$ at $T_{d 2}$ will be given by

$$
\left(\frac{T_{1}}{T_{2}}\right)^{3}=\frac{43}{4 N\left(T_{d 1}\right)}
$$

where $N\left(T_{d 1}\right)$ is the number of degrees of freedom at $T_{d 1}$ and the number of degrees of freedom at $T_{d 2}$ is $43 / 4$. Furthermore, the contribution to the number of neutrino degrees of freedom will be

$$
\Delta N_{\text {eff }}=\left(\frac{T_{1}}{T_{2}}\right)^{4}=\left(\frac{43}{4 N\left(T_{d 1}\right)}\right)^{4 / 3} .
$$

For example, the upper limit in Eq. (24), yields $T_{1} / T_{2}<$ 0.64 and $N\left(T_{d 1}\right)>162 / 4$ implying that the decoupling temperature should be greater than $\Lambda_{\mathrm{QCD}}$. That is decoupling should take place before the QCD transition in the early universe. Thus we arrive at an upper limit,

$$
\theta<\left(\frac{T_{d 2}}{T_{d 1}}\right)^{3 / 2}=\left(\frac{2 \mathrm{MeV}}{\Lambda_{\mathrm{QCD}}}\right)^{3 / 2} \lesssim 1.5 \times 10^{-3},
$$

for $\Lambda_{\mathrm{QCD}}=150 \mathrm{MeV}$.

As one can see, in the cosmologically viable region of interest, the value of $y_{s}$ (and $\theta$ ) are too weak to be constrained by $N_{\text {eff }}$ (at the $2 \sigma$ level). In contrast, the $1 \sigma$ upper limit to $N_{\text {eff }}$ is 0.05 [65], and in that case, $T_{1} / T_{2}<$ 0.47 and $N\left(T_{d 1}\right)>407 / 4$ implying that the decoupling temperature should be as large as $m_{t}$ (that is greater than all SM masses). In this case, the limit on $\theta$ is significantly stronger, $\theta<\left(2 \mathrm{MeV} / m_{t}\right)^{3 / 2} \approx 4 \times 10^{-8}$. Because the number of degrees of freedom varies slowly with temperature above $\Lambda_{\mathrm{QCD}}$ the limit on $\theta$ varies quickly with $\Delta N_{\text {eff }}$. At the value of $\theta \approx 1.5 \times 10^{-6}$ corresponding to $\alpha=0.05$, we would predict $T_{d 1} \approx 15 \mathrm{GeV}>m_{b}$, implying that $\Delta N_{\text {eff }} \approx 0.062$, which may be probed in future $\mathrm{CMB}$ missions. In other words, demanding that our model satisfies cosmological constraints therefore favours the region with $\alpha \sim 1$ which is more natural from the modelbuilding point of view.

\section{Results}

We note at this point that the combination of Eqs. (16) and (23) seem to point toward a natural region of the parameter space with $\alpha \simeq 10^{-2}, \theta \simeq 10^{-6}$, and $T_{\mathrm{RH}} \simeq$ $10^{11} \mathrm{GeV}$ which corresponds to $y_{\phi} \simeq y_{f} \simeq 10^{-5}$ from Eq. (19). In order to explore this region of the parameter space, we performed a scan on the set of parameters $\left\{y_{s}, y_{R}, m_{s}\right\}$, while fixing $M_{R}=10^{12} \mathrm{GeV}$ and requiring that $m_{2}=0.05 \mathrm{eV}$.

We show in Fig. 2 a scan of the plane $\left(\theta, m_{1} \approx m_{s}\right)$ after diagonalization of the mass matrix of Eq. (9). For all points considered, we have fixed the DM mass, $m_{a}=1 \mathrm{EeV}$, the 


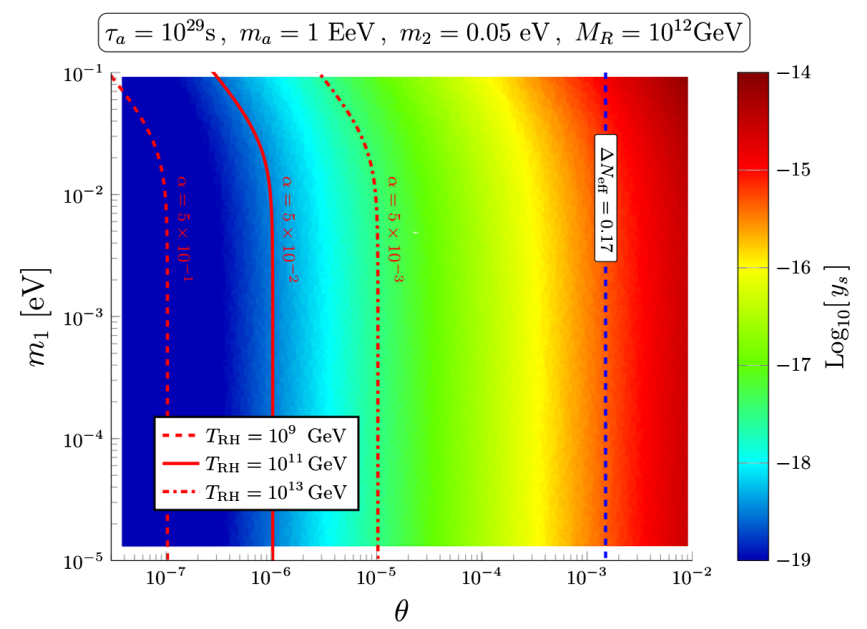

FIG. 2. Points in the $\left(\theta, m_{1}\right)$ parameter space for the case of an $\mathrm{EeV}$ dark matter candidate with a cosmological lifetime of $10^{29}$ seconds. The red lines correspond to three different values of $\alpha$ $\left(5 \times 10^{-1}, 5 \times 10^{-2}\right.$ and $\left.5 \times 10^{-3}\right)$ and their position in the plane is explained in the text.

DM lifetime, $\tau_{a}=10^{29} \mathrm{~s}$, the active neutrino mass, $m_{2}=$ $0.05 \mathrm{eV}$, and the inflaton mass, $m_{\Phi}=3 \times 10^{13} \mathrm{GeV}$. We consider three values of $\alpha$ as labeled. In the simple case where the inflaton couples equally to the sterile neutrino $\nu_{1}$ and SM fermions $\left(y_{\phi}=y_{f}\right)$, from Eq. (23) we can satisfy $\Omega_{a} h^{2} \simeq 0.12$ for different values of $\alpha$ by compensating with a different value the reheating temperature. For the values of $\alpha$ chosen, we require $T_{\mathrm{RH}}=10^{13}, 10^{11}$ and $10^{9} \mathrm{GeV}$ as indicated on the figure. The position of the lines of constant $\alpha$ is determined by setting the lifetime to the experimental limit of $\tau_{a}=10^{29} \mathrm{~s}$ which can be read from Eq. (16) (with $\left.\tau_{a} \approx \Gamma_{a \rightarrow \nu_{1} \nu_{2} h}^{-1}\right)$. Note that for $m_{1} \lesssim m_{2}$, the lifetime can be approximated by Eq. (17) which is independent of $m_{1}$ which explains why the lines are mostly vertical in the depicted plane. Moreover, since the lifetime is proportional to $(\alpha \theta)^{2}$, the choice of $\alpha$ determines $\theta$ for constant $\tau_{a}$. For each point of the scan, the value of the corresponding Yukawa coupling $y_{s}$ is indicated by the colored bar.

As was anticipated in the previous subsection, in the region of interest the correction to $\Delta N_{\text {eff }}$ is quite small as compared to the $95 \%$ CL upper limit $\Delta N_{\text {eff }}<0.17$, which corresponds to the dashed blue contour in Fig. 2.

We also see in the figure, that the coupling $y_{s}$ should be quite small $\left(\mathcal{O}\left(10^{-18}\right)\right.$ in the region of interest). We will justify this small coupling in Sec. V. Note that the reason the contribution of $\nu_{s}$ to $N_{\text {eff }}$ is small, is precisely because the coupling, $y_{s}$ (and mixing angle) is small.

\section{SIGNATURES}

\section{A. IceCube signals}

One clear signature of the model discussed above would be a monochromatic neutrino signal that could be observed by the IceCube, or the ANITA collaborations. In Ref. [51], the case of a scalar DM particle decaying into light righthanded neutrinos was studied and it was shown that the decay of an EeV DM particle followed by the scattering of the RH neutrino within the Earth's crust could lead to visible signals both for ANITA and IceCube for a mixing angle and DM lifetime of order $\tau_{a} / \theta^{2} \lesssim 10^{27} \mathrm{~s}$. As we have seen, the region of the parameter space that is favored in our model lies toward smaller values of the mixing angle $\theta \lesssim$ $10^{-5}$ and $\tau_{a} \gtrsim 10^{29} \mathrm{~s}$, leading to a ratio $\tau_{a} / \theta^{2} \gtrsim 10^{39} \mathrm{~s}$. This would indicate that our model cannot be detected in searches for anomalous upward-propagating cosmic rays. Similarly, in the $\mathcal{O}(1-10) \mathrm{EeV}$ range, EUSO-SPB2 [67] expects a sensitivity up to a hundred times better than ANITA, though that is also far below what would be needed in order to observe any upward-going signal.

In contrast, searches for downward-propagating ultrahigh-energy (UHE) cosmic rays are better suited for signatures of the model discussed here. The IceCube collaboration has reported limits on the decay of darkmatter particles with masses reaching up to a few hundred $\mathrm{PeV}$ to active neutrinos. Furthermore, it was shown in Refs. $[64,68]$ that the creation of electroweak showers from the decay of a heavy DM state into neutrinos at very high energy might be constrained at lower energy since the secondary products of such a shower might be visible in the form of a diffuse flux of neutrinos or photons at low energy. These studies led us in the previous sections to impose that the DM lifetime is larger than $\tau_{a} \gtrsim 10^{29} \mathrm{~s}$. Note, however, that future constraints on the flux of high-energy neutrinos in the TeV-PeV range, as expected from collaborations such as EUSO-SPB2 [67] or POEMMA [69] might help improving the limits derived in [64,68] and set more stringent constraints on our scenario. We leave such a study for future work.

In this section we determine the region of parameter space that might be probed experimentally by IceCube, either in the form of direct scattering of UHE neutrinos within the detector, or from secondary electroweak showers which would arrive on Earth at lower energies.

\section{B. Neutrino scattering in the IceCube detector}

Let us estimate the number of events which could be detected by IceCube under the form of a monochromatic neutrino signal at ultra-high energies. For that purpose, we suppose that the dark-matter particles follow a NavarroFrenk-White (NFW) profile [70]:

$$
\rho_{\mathrm{DM}}(r) \propto \frac{1}{\left(\frac{r}{r_{s}}\right)\left[1+\left(\frac{r}{r_{s}}\right)^{2}\right]},
$$

where $r_{s}=24 \mathrm{kpc}$ and the dark-matter density distribution is normalized to equal $\rho_{\odot}=0.3 \mathrm{GeV} \mathrm{cm}^{-3}$ in the vicinity of the solar system [71]. Following Ref. [51], the darkmatter flux, averaged over solid angle, is 


$$
\langle\Phi\rangle \simeq 1.6 \times 10^{-16} \mathrm{~cm}^{-2} \mathrm{~s}^{-1}\left(\frac{10^{29} \mathrm{~s}}{\tau_{\mathrm{DM}}}\right)\left(\frac{1 \mathrm{EeV}}{m_{\mathrm{DM}}}\right) .
$$

The number of events predicted for IceCube, assuming a fiducial volume of $\mathcal{V}_{\text {IC }} \approx(1 \mathrm{~km})^{3}$ and an exposure time of $\mathcal{T}_{\text {exp }}=3142.5$ days, is given by the relation

$$
N_{\text {events }}=\mathcal{V}_{\text {IC }} \cdot \rho_{\text {ice }} \cdot \mathcal{N}_{\mathrm{A}} \cdot \mathcal{T}_{\text {exp }} \cdot \sigma_{\nu N}\left(E_{\nu}\right),
$$

where the density of the ice is taken to be $\rho_{\text {ice }}=$ $0.92 \mathrm{~g} \mathrm{~cm}^{-3}, \mathcal{N}_{\mathrm{A}}$ is Avogadro's constant and we estimate the deep-inelastic scattering cross section $\sigma_{\nu N}$ of neutrinos scattering off nuclei using the results of Ref. [72]

$$
\log _{10}\left(\sigma_{\nu N}[E] / \mathrm{cm}^{2}\right)=\sum_{i=0}^{3} p_{i} \log _{10}(E / \mathrm{eV})^{i},
$$

with $\quad p_{0}=-53.5(-54.1), \quad p_{1}=2.66(2.65), \quad p_{2}=$ $-0.129(-0.112)$ and $p_{3}=0.00182(0.00175)$ for charged current (and neutral current) interactions, respectively. This yields the final result

$N_{\text {events }} \approx 0.2 \times\left(\frac{10^{29} \mathrm{~s}}{\tau_{a}}\right)\left(\frac{1 \mathrm{EeV}}{m_{a}}\right)\left(\frac{\sigma_{\nu N}\left[m_{a} / 2\right]}{2.6 \times 10^{-33} \mathrm{~cm}^{2}}\right)$.

Therefore, in the region of the parameter space which we have considered, $\tau_{a} \gtrsim 10^{29} \mathrm{~s}$, the number of events that IceCube might see within the detector is expected to be of order one. Therefore, it is reasonable to suppose that increasing the exposure time by a factor of a few could lead to the detection of such signal in the relatively near future.

\section{Secondary electroweak shower detection}

In Ref. [64], limits on the lifetime of a dark-matter particle decaying into active neutrinos have been derived from IceCube data by studying the secondary showers that would be produced by electroweak states at lower energies. We used the limit of Ref. [64] on the lifetime $\tau_{a}$ as a function of the dark-matter mass $m_{a}$ in order to translate it into a limit on the mixing angle $\theta$ for a fixed set of parameters.

\section{Results}

Our results are summarized in Fig. 3 where we have fixed the value of $\alpha$ to one of our previous benchmark points, $\alpha=5 \times 10^{-2}$, and fixed the active neutrino mass to be $m_{2}=0.05 \mathrm{eV} \gg m_{1}$. The green-shaded area indicates parameter values in the $m_{a}-\theta$ plane for which the lifetime of dark matter would be shorter than the age of the universe. This occurs only at large values of both $m_{a}$ and $\theta$ and would be excluded by the lack of events at IceCube. The

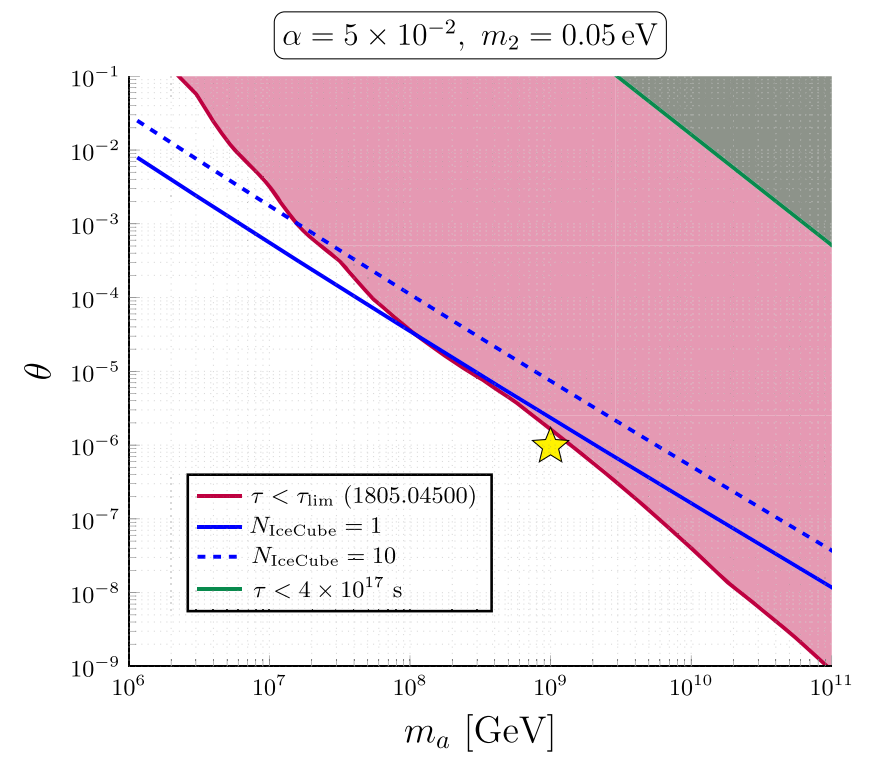

FIG. 3. Regions of the $m_{a}-\theta$ parameter plane that are excluded by astrophysical constraints obtained by neutrino detectors (shaded purple). In the green shaded region, the lifetime of the DM candidate is shorter than the age of the Universe. Along the solid (dashed) blue line, the number of events expected by Icecube in its exposure time is 1 (10). The yellow star indicates the benchmark point $m_{a}=1 \mathrm{EeV}$ and $\theta \simeq 1.5 \times 10^{-6}$.

purple-shaded area excludes the region of the parameter space in which the lifetime of dark matter is shorter than the limit derived in Ref. [64]. Finally the blue lines indicate the value of the mixing angle $\theta$ as a function of the DM mass, $m_{a}$ which would lead to 1 event (solid line) or 10 events (dashed line) in the IceCube detector given the exposure time $\mathcal{T}_{\text {exp }}=3142.5$ days. As one can see, our benchmark point (yellow star) corresponding to $\alpha=5 \times 10^{-2}, m_{2}=$ $0.05 \mathrm{eV} \gg m_{1}$ with $m_{a}=1 \mathrm{EeV}$ and $\tau_{a}=10^{29} \mathrm{~s}$ (corresponding to $\theta \simeq 1.5 \times 10^{-6}$ ) is flirting with the experimental limits we presented above, suggesting that the prospect for discovery or exclusion of this benchmark is quite high for IceCube, especially for dark-matter masses ranging from the PeV scales to $\mathrm{EeV}$ scales.

As we have seen, current IceCube data is already on the edge of discovery of $\mathrm{EeV}$ dark matter. In its next phase, starting next year, the IceCube collaboration will be able to probe the $\mathrm{EeV}$ scale with much better sensitivity for an observable signal.

\section{TOWARD A MICROSCOPIC APPROACH}

In this section we develop a toy microscopic model that could justify our assumed hierarchy given in Eq. (10). In fact, such a hierarchy can be generated naturally by the spontaneous breaking of a global $U(1)$ symmetry and the generation of nonrenormalizable operators at low-energy. We introduce a set of heavy Weyl fermion pairs $\tilde{\psi}_{i}, \psi_{i}$ with $i=1,4$ and a complex scalar field $S$ whose charges are 

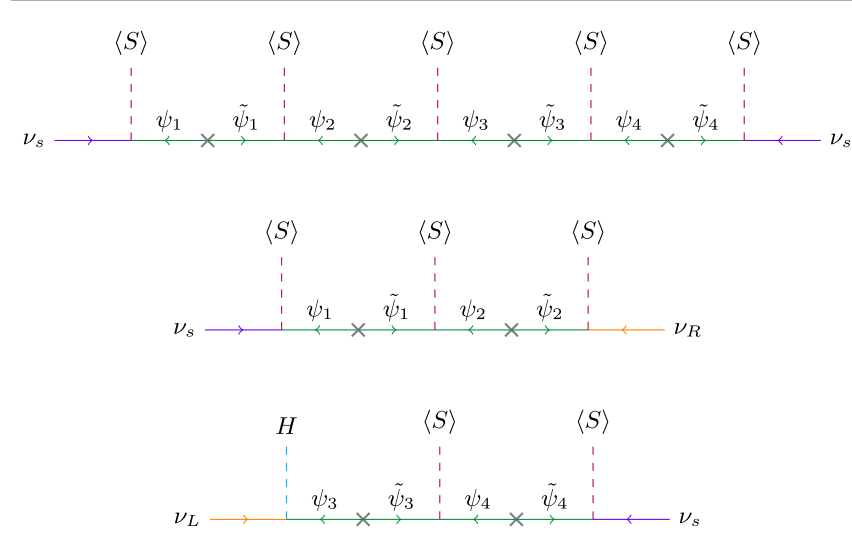

FIG. 4. Feynman diagrams involving the heavy fermions $\left\{\psi_{i}, \tilde{\psi}_{i}\right\}_{i=1,4}$, the scalar $S$ and the different neutrino species.

given in Table I. One can integrate out these heavy fermions and obtain effective interactions between the scalar $S$ and the different neutrino species, as can be seen from Fig. 4.

Assuming that the fermions $\left\{\psi_{i}, \tilde{\psi}_{i}\right\}_{i=1,4}$ have masses of the same order of magnitude $M_{i} \sim M$ where $M$ is some mass scale close to the Planck scale, after integrating out the heavy fermions, one obtains the effective low energy the Lagrangian

$$
\begin{aligned}
\mathcal{L}_{U(1)}^{\mathrm{eff}} \supset & \frac{\alpha}{M_{P}} \partial_{\mu} a \bar{\nu}_{s} \gamma^{\mu} \gamma_{5} \nu_{s}-\left(h_{S} \frac{S^{5}}{M^{4}} \bar{\nu}_{S}^{c} \nu_{s}+h_{R} S \bar{\nu}_{R}^{c} \nu_{R}\right. \\
& +h_{S R} \frac{S^{3}}{M^{2}} \bar{\nu}_{s}^{c} \nu_{R}+h_{L S} \frac{S^{2}}{M^{2}} \tilde{H} \bar{L}_{L} \nu_{s} \\
& \left.+\lambda_{L}^{R} \tilde{H} \bar{L}_{L} \nu_{R}+\text { H.c. }\right)
\end{aligned}
$$

in four-component notation. In the above expression, we have introduced the effective couplings

$$
\begin{aligned}
& h_{S}=\lambda_{4}^{S} \lambda_{3}^{4} \lambda_{2}^{3} \lambda_{1}^{2} \lambda_{S}^{1}, \quad h_{S R}=\lambda_{S}^{1} \lambda_{1}^{2} \lambda_{2}^{R}, \\
& h_{R}=\lambda_{R} \quad \text { and } \quad h_{L S}=\lambda_{L}^{3} \lambda_{3}^{4} \lambda_{4}^{S},
\end{aligned}
$$

where the microscopic couplings $\lambda_{i}$ are defined in Appendix B.

We assume that the global $U(1)$ symmetry is broken spontaneously at some high energy scale, and the scalar field acquires a vacuum expectation value $\langle S\rangle \neq 0$. After spontaneous symmetry breaking, one obtains the low energy Lagrangian

$$
\begin{aligned}
\mathcal{L}_{L(H)}^{\text {eff }} & \supset \frac{\alpha}{M_{P}} \partial_{\mu} a \bar{\nu}_{s} \gamma^{\mu} \gamma_{5} \nu_{s}-\left(\frac{1}{2} m_{s} \bar{\nu}_{s}^{c} \nu_{s}+\frac{1}{2} M_{R} \bar{\nu}_{R}^{c} \nu_{R}\right. \\
& \left.+\frac{1}{2} m_{S R} \bar{\nu}_{s}^{c} \nu_{R}+y_{s} \tilde{H} \bar{L} \nu_{s}+y_{R} \tilde{H} \bar{L}_{L} \nu_{R}+\text { H.c. }\right),
\end{aligned}
$$

where we defined
TABLE I. Charge assignment of the UV particle content under the new global $U(1)$ symmetry.

$$
\begin{aligned}
& \begin{array}{llllllllllllll}
\hline a & L & S & \nu_{S} & \nu_{R} & \psi_{1} & \tilde{\psi}_{1} & \psi_{2} & \tilde{\psi}_{2} & \psi_{3} & \tilde{\psi}_{3} & \psi_{4} & \tilde{\psi}_{4} \\
\hline
\end{array} \\
& \begin{array}{llllllllllllll}
\hline U(1) & 0 & -1 & -2 & +5 & +1 & -3 & 3 & -1 & 1 & 1 & -1 & 3 & -3 \\
\hline
\end{array} \\
& \begin{array}{rlrl}
m_{s} & \equiv 2 h_{S} \frac{\langle S\rangle^{5}}{M^{4}}, & M_{R} \equiv 2 h_{R}\langle S\rangle, \\
y_{s} \equiv h_{L S} \frac{\langle S\rangle^{2}}{M^{2}}, & y_{R} \equiv \lambda_{L}^{R} .
\end{array}
\end{aligned}
$$

If we assume all of the couplings $\lambda_{i}^{j} \sim 0.1$, with the exception of $\lambda_{L}^{3}$ which we take to be $\sim 10^{-4}$, and a heavy mass scale $M \simeq M_{P}$ and a symmetry breaking scale of

$$
\langle S\rangle \simeq 5 \times 10^{12} \mathrm{GeV},
$$

we naturally get the desired hierarchy of scales

$$
\begin{array}{rlrl}
m_{s} & \approx 2 \times 10^{-6} \mathrm{eV} & M_{R} \approx 10^{12} \mathrm{GeV} \\
y_{s} & \approx 4 \times 10^{-18} & y_{R} & \sim 0.1,
\end{array}
$$

which approximates the favored parameter space of our model. Note that in addition to the model we have previously studied, there is an additional mixing term $h_{S R} \frac{S^{3}}{M_{p}^{2}} \bar{\nu}_{s}^{c} \nu_{R}$ in the seesaw mass matrix, but we checked that for $h_{S R} \sim 10^{-3}$ this term does not perturb the seesaw mechanism or our mass hierarchy.

Due to the charge assignment, a coupling of the inflaton of the type $\Phi \bar{\nu}_{s}^{c} \nu_{s}$ in Eq. (6) is not allowed because of the neutrality of $\Phi$ under this $U(1)$. It is then impossible to generate a sufficiently large quantity of dark matter through the decay process shown in Fig. 1. However, the production of dark matter via the 3-body decay of the inflaton could be made possible by considering a term like $\mu_{\Phi} \Phi|S|^{2}$, with $\mu_{\Phi}$ being a dimensionful parameter in addition to the term $h_{R} S \bar{\nu}_{R}^{c} \nu_{R}$ with $h_{R} \sim 0.1$, as depicted in Fig. 5. In this case,

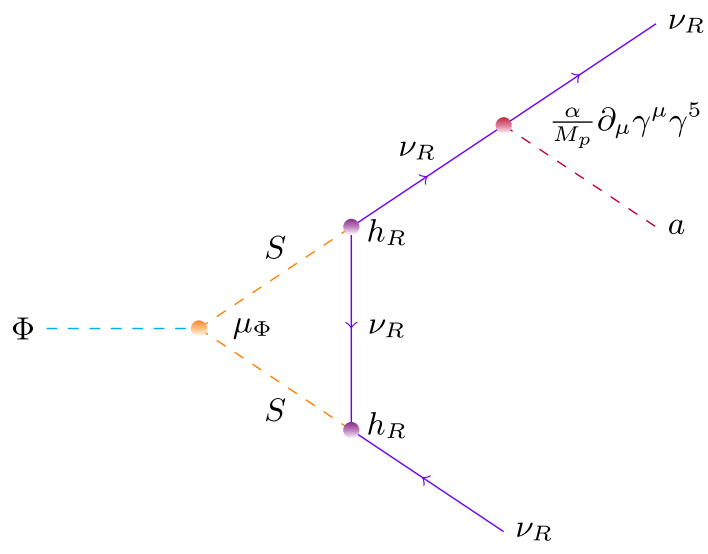

FIG. 5. Inflaton decay process, source of the dark matter abundance in the microscopic model. 
we expect the effective inflaton decay coupling to be $y_{\phi} \sim \mu_{\Phi} h_{R}^{2} f\left(M_{S} / M_{R}\right) /\left(16 \pi^{2} M_{R}\right) \sim 10^{-5}$ for $h_{R} \sim 0.1$, $\mu_{\Phi} / M_{R} \sim 0.1$ and $f\left(M_{S} / M_{R}\right) \sim 1$ if $M_{S} \sim M_{R}$ as we expect, $M_{S}$ being the mass of the heavy scalar state.

\section{CONCLUSION}

The most commonly considered mass ranges for dark matter have been either WIMPs with masses between $100 \mathrm{GeV}$ to $1 \mathrm{TeV}$, or axions with masses much less than an $\mathrm{eV}$. Despite a vigorous search program neither have yet been discovered. Therefore it is natural to open up the possible mass range for new searches for dark matter. Indeed there is a lot of effort going into sub-GeV candidates and the prospects for new direct detection experiments. Here we have explored another regime of dark matter masses of an EeV.

In this paper, we have shown that we can reconcile the dark matter lifetime, which requires extremely reduced couplings, with a natural production mechanism. The long lifetime is possible when Planck-mass suppressed operators (generated, for example, by the breaking of a global symmetry) are combined with tiny neutrino masses. The induced lifetime respects the strongest indirect detection limits once the dark matter is coupled to the neutrino sector. Moreover, despite the feebleness of the coupling, we showed that inflaton decay into dark matter can be sufficient to produce a relic abundance compatible with PLANCK results. Furthermore, we showed that the next generation of neutrino telescopes will be able to probe such heavy dark matter in the near future.

\section{ACKNOWLEDGMENTS}

The authors want to thank especially Marcos Garcia for very insightful discussions. This work was supported in part by the France-US PICS MicroDark and the ANR Grant No. Black-dS-String ANR-16-CE31-0004-01. The work of M. P. was supported by the Spanish Agencia Estatal de Investigación through the Grants No. FPA2015-65929-P (MINECO/FEDER, UE), No. PGC2018-095161-B-I00, IFT Centro de Excelencia Severo Ochoa SEV-20160597, and Red Consolider MultiDark FPA2017-90566REDC. The research activities of L. H. are supported in part by the Department of Energy under Grant No. DE-FG0213ER41976(de-sc0009913). This work was partially performed at the Aspen Center for Physics, which is supported by National Science Foundation Grant No. PHY-1607611. The work of LH has been partially performed during the workshop "Dark Matter as a Portal to New Physics" supported by Asia Pacific Center for Theoretical Physics. K. O. and M.P. would like to thank the Lawrence Berkeley National Laboratory for its hospitality during part of the realization of this work. M. P. would like to thank the Université Libre de Bruxelles for its hospitality during the last stages of the realization of this work. M. P. and L. H. would also like to thank the Paris-Saclay Particle Symposium with the support of the P2I and SPU research departments and the P2IO Laboratory of Excellence (program "Investissements d'avenir" ANR-11-IDEX-0003-01 Paris-Saclay and ANR-10-LABX-0038), as well as the IPhT. This project has received funding/support from the European Unions Horizon 2020 research and innovation programme under the Marie Skodowska-Curie grant agreements Elusives ITN No. 674896 and InvisiblesPlus RISE No. 690575. The work of K. O. was supported in part by the DOE Grant No. DE-SC0011842 at the University of Minnesota.

\section{APPENDIX A: DECAY RATES}

In this appendix, we provide some relevant details concerning the computation of the dark matter decay rate. From Eq. (14), we see that up to $\mathcal{O}\left(\theta^{2}\right)$ there are two twobody final state decay channels to consider

$$
\begin{gathered}
\Gamma_{a \rightarrow \nu_{1} \nu_{1}}=\frac{\alpha^{2} m_{a} m_{1}^{2}}{\pi M_{P}^{2}} \\
\Gamma_{a \rightarrow \nu_{1} \nu_{2}} \simeq \frac{\alpha^{2} \theta^{2} m_{a}\left(m_{1}+m_{2}\right)^{2}}{2 \pi M_{P}^{2}}
\end{gathered}
$$

where we neglected some threshold factors which are negligible in the limit $m_{a} \gg m_{2}, m_{1}$. The direct decay of $a$ to two SM-like neutrinos is suppressed by $\theta^{4}$.

There are also three-body final state decays which involve a Higgs, $W^{ \pm}$, or $Z$ in the final state. These couple to neutrinos in the $\nu_{1}, \nu_{2}$ basis through

$$
\begin{aligned}
\mathcal{L}= & -\frac{g}{4 c_{W}} Z^{\mu}\left(\bar{\nu}_{2} \gamma_{\mu} \gamma_{5} \nu_{2}\right. \\
& \left.+\theta\left(\bar{\nu}_{2} \gamma_{\mu} \gamma_{5} \nu_{1}+\bar{\nu}_{1} \gamma_{\mu} \gamma_{5} \nu_{2}\right)+\mathcal{O}\left(\theta^{2}\right)\right) \\
& -\frac{g}{\sqrt{2}}\left(\bar{N}_{2} \gamma^{\mu} e_{L} W_{\mu}^{+}+\bar{e}_{L} \gamma^{\mu} N_{2} W_{\mu}^{-}\right. \\
& \left.+\theta\left(\bar{\nu}_{1} \gamma^{\mu} e_{L} W_{\mu}^{+}+\bar{e}_{L} \gamma^{\mu} \nu_{1} W_{\mu}^{-}\right)+\mathcal{O}\left(\theta^{2}\right)\right) \\
& -y_{S} \frac{h}{2 \sqrt{2}}\left(\bar{\nu}_{2} \nu_{1}+\bar{\nu}_{1} \nu_{2}\right. \\
& \left.+2 \theta\left(\bar{\nu}_{1} \nu_{1}-\bar{\nu}_{2} \nu_{2}\right)+\mathcal{O}\left(\theta^{2}\right)\right)
\end{aligned}
$$

where we used $H=\left(v_{h}+h\right) / \sqrt{2}$ in unitary gauge, where $h$ is the Higgs real scalar field. This leads to the three-body decay width with a Higgs in the final which is given by

$\Gamma_{a \rightarrow \nu_{1} \nu_{2} h}=\frac{\alpha^{2} m_{a}^{3} y_{S}^{2}}{384 \pi^{3} M_{P}^{2}}=\frac{\alpha^{2} m_{a}^{3} \theta^{2}}{192 \pi^{3} v_{h}^{2} M_{P}^{2}}\left(m_{1}+m_{2}\right)^{2}$

where we used the relation between the Yukawa coupling and the mixing angle. Similarly, 


$$
\Gamma_{a \rightarrow \nu_{1} \nu_{2} Z}=\frac{\alpha^{2} e^{2} \theta^{2} m_{a}^{3}}{768 \pi^{3} M_{P}^{2} c_{W}^{2} m_{Z}^{2} s_{W}^{2}}\left(m_{2}-m_{1}\right)^{2},
$$

where we assumed $m_{1} \sim m_{2} \ll m_{Z} \ll m_{a}$ and $e=$ $g g^{\prime} / \sqrt{g^{2}+g^{\prime 2}}$ is the electromagnetic coupling constant. Assuming the hierarchy $m_{2} \sim m_{1} \ll m_{e} \ll m_{W} \ll m_{a}$, gives

$$
\Gamma_{a \rightarrow \nu_{1} e_{L} W}=\frac{\alpha^{2} g^{2} \theta^{2} m_{a}^{3} m_{1}^{2}}{768 \pi^{3} M_{P}^{2} m_{W}^{2}},
$$

which is of the same order than the partial width $\Gamma_{a \rightarrow \nu_{1} \nu_{2} Z}$ by using relations between couplings and weak boson masses.

As we have seen, the absence of a helicity flip in the case of the three-body decay compensates the higher power of the Yukawa coupling which arises in the decay width. Thus the ratio of the 3- to 2-body decay widths is

$$
\begin{aligned}
\frac{\Gamma_{a \rightarrow \nu_{1} \nu_{2} h}}{\Gamma_{a \rightarrow \nu_{1} \nu_{1}}} & =\frac{m_{a}^{2} \theta^{2}}{192 \pi^{2} v_{h}^{2}} \frac{\left(m_{1}+m_{2}\right)^{2}}{m_{1}^{2}} \\
& \gtrsim\left(\frac{m_{2}}{m_{1}}\right)^{2}\left(\frac{\theta}{10^{-5}}\right)^{2}\left(\frac{m_{a}}{\mathrm{EeV}}\right)^{2},
\end{aligned}
$$

and both 2- and 3-body decay modes could be relevant depending on the value of $\theta$ and the ratio of light neutrino masses. However, the 3-body decay always dominates over the 2-body decay when SM particles are in the final state

$$
\frac{\Gamma_{a \rightarrow N_{1} N_{2} h}}{\Gamma_{a \rightarrow N_{2} N_{1}}}=\frac{m_{a}^{2}}{96 \pi^{2} v_{h}^{2}} \gg 1
$$

Finally, when going to 4-body and higher decay processes, the major change in the decay width, besides complexifying the phase space volume, is an increase in the powers of the Yukawa coupling

$$
\Gamma_{4-\text { body }} \sim \frac{\alpha^{2} y_{S}^{4}}{M_{P}^{2}} m_{a}^{3} \Rightarrow \frac{\Gamma_{4-\text { body }}}{\Gamma_{3-\text { body }}} \sim y_{S}^{2} .
$$

which naturally leads to smaller widths than the 3-body decay modes.

\section{APPENDIX B: THE MICROSCOPIC MODEL}

We provide here the microscopic Lagrangian which allows us to derive the $U(1)$ invariant effective theory in Eq. (33) of Section V. Using a two-component notation, the most general renormalizable and $U(1)$ invariant Lagrangian that one can write involving the fields of the model in Sec. V is

$$
\begin{aligned}
\mathcal{L}_{\mathrm{UV}} \supset & -\left(\lambda_{S}^{1} S \nu_{s} \psi_{1}+\lambda_{1}^{2} S \tilde{\psi}_{1} \psi_{2}+\lambda_{2}^{3} S \tilde{\psi}_{2} \psi_{3}+\lambda_{3}^{4} \tilde{\psi}_{3} \psi_{4}\right. \\
& +\lambda_{4}^{S} S \tilde{\psi}_{4} \nu_{s}+\lambda_{2}^{R} S \tilde{\psi}_{2} \nu_{R}+\lambda_{R} S \nu_{R} \nu_{R}+\lambda_{L}^{3} \tilde{H} L_{L} \psi_{3} \\
& \left.+\lambda_{L}^{R} \tilde{H} L_{L} \nu_{R}+\text { H.c. }\right)-\sum_{i=1}^{4} M_{i} \tilde{\psi}_{i} \psi_{i}-V(S) \\
& +\frac{\alpha}{M_{P}} \partial_{\mu} a\left(\bar{\nu}_{s} \bar{\sigma}^{\mu} \nu_{s}+\bar{\nu}_{R} \bar{\sigma}^{\mu} \nu_{R}\right) .
\end{aligned}
$$

Taking a common mass $M_{i} \equiv M$ for all the supermassive fermions $\left\{\psi_{i}, \tilde{\psi}_{i}\right\}_{i=1,4}$, one can integrate them out to obtain the effective operators

$$
\begin{aligned}
\mathcal{L}_{\text {eff }} \supset & -\frac{\lambda_{4}^{S} \lambda_{3}^{4} \lambda_{2}^{3} \lambda_{1}^{2} \lambda_{S}^{1}}{M^{4}} S^{5} \nu_{s} \nu_{s} \\
& -\frac{\lambda_{2}^{R} \lambda_{1}^{2} \lambda_{S}^{1}}{M^{2}} S^{3} \nu_{R} \nu_{s}-\frac{\lambda_{L}^{3} \lambda_{3}^{4} \lambda_{4}^{S}}{M^{2}} S^{2} \tilde{H} L_{L} \nu_{s}+\text { H.c. }
\end{aligned}
$$

One thereafter obtains at low energy the Lagrangian of Eq. (33), written using four-component notation

$\nu_{s} \rightarrow\left(\begin{array}{c}\nu_{s} \\ 0\end{array}\right), \quad \nu_{R} \rightarrow\left(\begin{array}{c}\nu_{R} \\ 0\end{array}\right), \quad \nu_{L} \rightarrow\left(\begin{array}{c}0 \\ \bar{\nu}_{L}\end{array}\right)$,

and introducing the effective couplings of Eq. (34).

In full generality, certain interaction or mass terms could be added to the Lagrangian of Eq. (B1) while preserving the symmetries of the model. However, we checked that the presence of such terms do not modify the structure of the effective theory we introduce in Sec. V but simply generate additional contributions to the relations of Eq. (34).
[1] P. A. R. Ade et al. (Planck Collaboration), Astron. Astrophys. 594, A13 (2016); N. Aghanim et al. (Planck Collaboration), arXiv:1807.06209.

[2] E. Aprile et al. (XENON Collaboration), Phys. Rev. Lett. 121, 111302 (2018).

[3] D. S. Akerib et al. (LUX Collaboration), Phys. Rev. Lett. 118, 021303 (2017).
[4] X. Cui et al. (PandaX-II Collaboration), Phys. Rev. Lett. 119, 181302 (2017).

[5] M. Ackermann et al. (Fermi-LAT Collaboration), Phys. Rev. D 91, 122002 (2015); 89, 042001 (2014); A. Albert et al. (FermiLAT and DES Collaborations), Astrophys. J. 834, 110 (2017).

[6] L. Rinchiuso (H.E.S.S. Collaboration), EPJ Web Conf. 209, 01023 (2019). 
[7] M. L. Ahnen et al. (MAGIC and Fermi-LAT Collaborations), J. Cosmol. Astropart. Phys. 02 (2016) 039.

[8] M. G. Aartsen et al. (IceCube Collaboration), Eur. Phys. J. C 78, 831 (2018).

[9] P. W. Gorham et al. (ANITA Collaboration), Phys. Rev. Lett. 117, 071101 (2016); 121, 161102 (2018).

[10] V. Silveira and A. Zee, Phys. Lett. B 161, 136 (1985); J. McDonald, Phys. Rev. D 50, 3637 (1994); C. P. Burgess, M. Pospelov, and T. ter Veldhuis, Nucl. Phys. B619, 709 (2001); H. Davoudiasl, R. Kitano, T. Li, and H. Murayama, Phys. Lett. B 609, 117 (2005); H. Han and S. Zheng, J. High Energy Phys. 12 (2015) 044.

[11] J. A. Casas, D. G. Cerdeño, J. M. Moreno, and J. Quilis, J. High Energy Phys. 05 (2017) 036; A. Djouadi, O. Lebedev, Y. Mambrini, and J. Quevillon, Phys. Lett. B 709, 65 (2012); A. Djouadi, A. Falkowski, Y. Mambrini, and J. Quevillon, Eur. Phys. J. C 73, 2455 (2013); O. Lebedev, H. M. Lee, and Y. Mambrini, Phys. Lett. B 707, 570 (2012); Y. Mambrini, Phys. Rev. D 84, 115017 (2011).

[12] J. Ellis, A. Fowlie, L. Marzola, and M. Raidal, Phys. Rev. D 97, 115014 (2018); G. Arcadi, Y. Mambrini, and F. Richard, J. Cosmol. Astropart. Phys. 03 (2015) 018; J. Kearney, N. Orlofsky, and A. Pierce, Phys. Rev. D 95, 035020 (2017); M. Escudero, A. Berlin, D. Hooper, and M. X. Lin, J. Cosmol. Astropart. Phys. 12 (2016) 029.

[13] A. Alves, S. Profumo, and F. S. Queiroz, J. High Energy Phys. 04 (2014) 063; O. Lebedev and Y. Mambrini, Phys. Lett. B 734, 350 (2014); G. Arcadi, Y. Mambrini, M. H. G. Tytgat, and B. Zaldivar, J. High Energy Phys. 03 (2014) 134; O. Lebedev and Y. Mambrini, Phys. Lett. B 734, 350 (2014).

[14] H. P. Nilles, Phys. Rep. 110, 1 (1984); H. E. Haber and G. L. Kane, Phys. Rep. 117, 75 (1985).

[15] H. Goldberg, Phys. Rev. Lett. 50, 1419 (1983).

[16] J. Ellis, J. Hagelin, D. Nanopoulos, K. Olive, and M. Srednicki, Nucl. Phys. B238, 453 (1984).

[17] J. Ellis, J. L. Evans, F. Luo, N. Nagata, K. A. Olive, and P. Sandick, Eur. Phys. J. C 76, 8 (2016); J. Ellis, J. L. Evans, F. Luo, K. A. Olive, and J. Zheng, Eur. Phys. J. C 78, 425 (2018); E. Bagnaschi et al., Eur. Phys. J. C 79, 149 (2019); J. Ellis, J. L. Evans, N. Nagata, K. A. Olive, and L. VelascoSevilla, Eur. Phys. J. C 80, 332 (2020).

[18] E. A. Bagnaschi et al., Eur. Phys. J. C 75, 500 (2015).

[19] P. Bechtle et al., Eur. Phys. J. C 76, 96 (2016).

[20] M. Aaboud et al. (ATLAS Collaboration), J. High Energy Phys. 06 (2018) 107; Phys. Rev. D 97, 112001 (2018); A. M. Sirunyan et al. (CMS Collaboration), Eur. Phys. J. C 77, 710 (2017); J. High Energy Phys. 05 (2018) 025.

[21] G. Arcadi, M. Dutra, P. Ghosh, M. Lindner, Y. Mambrini, M. Pierre, S. Profumo, and F. S. Queiroz, Eur. Phys. J. C 78, 203 (2018).

[22] H. Pagels and J. R. Primack, Phys. Rev. Lett. 48, 223 (1982); D. V. Nanopoulos, K. A. Olive, and M. Srednicki, Phys. Lett. 127B, 30 (1983); M. Y. Khlopov and A. D. Linde, Phys. Lett. 138B, 265 (1984); K. A. Olive, D. N. Schramm, and M. Srednicki, Nucl. Phys. B255, 495 (1985); J. R. Ellis, K. A. Olive, Y. Santoso, and V. C. Spanos, Phys. Lett. B 588, 7 (2004); J. L. Feng, S. f. Su, and F. Takayama, Phys. Rev. D 70, 063514 (2004); J. L. Feng, S. Su, and F. Takayama, Phys. Rev. D 70, 075019 (2004); F. D. Steffen,
J. Cosmol. Astropart. Phys. 09 (2006) 001; W. Buchmuller, L. Covi, K. Hamaguchi, A. Ibarra, and T. Yanagida, J. High Energy Phys. 03 (2007) 001; W. Buchmuller, AIP Conf. Proc. 1200, 155 (2010); S. Bailly, K. Y. Choi, K. Jedamzik, and L. Roszkowski, J. High Energy Phys. 05 (2009) 103; L. Covi, J. Hasenkamp, S. Pokorski, and J. Roberts, J. High Energy Phys. 11 (2009) 003.

[23] L. J. Hall, K. Jedamzik, J. March-Russell, and S. M. West, J. High Energy Phys. 03 (2010) 080; X. Chu, T. Hambye, and M. H. G. Tytgat, J. Cosmol. Astropart. Phys. 05 (2012) 034; X. Chu, Y. Mambrini, J. Quevillon, and B. Zaldivar, J. Cosmol. Astropart. Phys. 01 (2014) 034; A. Biswas, D. Borah, and A. Dasgupta, Phys. Rev. D 99, 015033 (2019).

[24] N. Bernal, M. Heikinheimo, T. Tenkanen, K. Tuominen, and V. Vaskonen, Int. J. Mod. Phys. A 32, 1730023 (2017).

[25] L. Heurtier and F. Huang, Phys. Rev. D 100, 043507 (2019); A. Berlin, D. Hooper, and G. Krnjaic, Phys. Rev. D 94, 095019 (2016); Phys. Lett. B 760, 106 (2016); M. Heikinheimo, T. Tenkanen, K. Tuominen, and V. Vaskonen, Phys. Rev. D 94, 063506 (2016); 96, 109902(E) (2017).

[26] Y. Mambrini, K. A. Olive, J. Quevillon, and B. Zaldivar, Phys. Rev. Lett. 110, 241306 (2013); N. Nagata, K. A. Olive, and J. Zheng, J. High Energy Phys. 10 (2015) 193; Y. Mambrini, N. Nagata, K. A. Olive, and J. Zheng, Phys. Rev. D 93, 111703 (2016); X. Chu, Y. Mambrini, J. Quevillon, and B. Zaldivar, J. Cosmol. Astropart. Phys. 01 (2014) 034.

[27] Y. Mambrini, N. Nagata, K. A. Olive, J. Quevillon, and J. Zheng, Phys. Rev. D 91, 095010 (2015); N. Nagata, K. A. Olive, and J. Zheng, J. Cosmol. Astropart. Phys. 02 (2017) 016.

[28] G. Bhattacharyya, M. Dutra, Y. Mambrini, and M. Pierre, Phys. Rev. D 98, 035038 (2018).

[29] N. Bernal, M. Dutra, Y. Mambrini, K. Olive, M. Peloso, and M. Pierre, Phys. Rev. D 97, 115020 (2018).

[30] K. Benakli, Y. Chen, E. Dudas, and Y. Mambrini, Phys. Rev. D 95, 095002 (2017).

[31] E. Dudas, Y. Mambrini, and K. Olive, Phys. Rev. Lett. 119, 051801 (2017).

[32] E. Dudas, T. Gherghetta, Y. Mambrini, and K. A. Olive, Phys. Rev. D 96, 115032 (2017).

[33] E. Dudas, T. Gherghetta, K. Kaneta, Y. Mambrini, and K. A. Olive, Phys. Rev. D 98, 015030 (2018).

[34] S. A. R. Ellis, T. Gherghetta, K. Kaneta, and K. A. Olive, Phys. Rev. D 98, 055009 (2018).

[35] K. Kaneta, Y. Mambrini, and K. A. Olive, Phys. Rev. D 99, 063508 (2019).

[36] D. Chowdhury, E. Dudas, M. Dutra, and Y. Mambrini, Phys. Rev. D 99, 095028 (2019).

[37] G. F. Giudice, E. W. Kolb, and A. Riotto, Phys. Rev. D 64, 023508 (2001); D. J. H. Chung, E. W. Kolb, and A. Riotto, Phys. Rev. D 60, 063504 (1999).

[38] M. A. G. Garcia, Y. Mambrini, K. A. Olive, and M. Peloso, Phys. Rev. D 96, 103510 (2017).

[39] M. A. G. Garcia and M. A. Amin, Phys. Rev. D 98, 103504 (2018).

[40] G. R. Farrar and P. Fayet, Phys. Lett. 76B, 575 (1978).

[41] M. De Montigny and M. Masip, Phys. Rev. D 49, 3734 (1994).

[42] S. W. Hawking, Commun. Math. Phys. 43, 199 (1975); 46, 206(E) (1976). 
[43] S. W. Hawking, D. N. Page, and C. N. Pope, Phys. Lett. 86B, 175 (1979); M. J. Perry, Phys. Rev. D 19, 1720 (1979); S. Giddings and A. Strominger, Nucl. Phys. B307, 854 (1988); J. Ellis and M. K. Gaillard, Phys. Lett. 88B, 315 (1979); R. Barbieri, J. Ellis, and M. K. Gaillard, Phys. Lett. 90B, 249 (1980).

[44] R. Kallosh, A. D. Linde, D. A. Linde, and L. Susskind, Phys. Rev. D 52, 912 (1995).

[45] T. Banks, M. Johnson, and A. Shomer, J. High Energy Phys. 09 (2006) 049.

[46] Y. Mambrini, S. Profumo, and F. S. Queiroz, Phys. Lett. B 760, 807 (2016).

[47] S. Profumo, F. Queiroz, and C. Siqueira, arXiv:1903.07638; C. Boehm, D. Hooper, J. Silk, M. Casse, and J. Paul, Phys. Rev. Lett. 92, 101301 (2004).

[48] Y. Mambrini and T. Toma, Eur. Phys. J. C 75, 570 (2015); E. Dudas, L. Heurtier, and Y. Mambrini, Phys. Rev. D 90, 035002 (2014); A. Boyarsky, J. Franse, D. Iakubovskyi, and O. Ruchayskiy, Phys. Rev. Lett. 115, 161301 (2015); H. M. Lee, Phys. Lett. B 738, 118 (2014); Y. Farzan and A. R. Akbarieh, J. Cosmol. Astropart. Phys. 11 (2014) 015.

[49] A. Esmaili and P. D. Serpico, J. Cosmol. Astropart. Phys. 11 (2013) 054; K. Murase, R. Laha, S. Ando, and M. Ahlers, Phys. Rev. Lett. 115, 071301 (2015); C. El Aisati, M. Gustafsson, and T. Hambye, Phys. Rev. D 92, 123515 (2015); B. Feldstein, A. Kusenko, S. Matsumoto, and T. T. Yanagida, Phys. Rev. D 88, 015004 (2013); L. Covi, M. Grefe, A. Ibarra, and D. Tran, J. Cosmol. Astropart. Phys. 04 (2010) 017; Y. Sui and P. S. Bhupal Dev, J. Cosmol. Astropart. Phys. 07 (2018) 020.

[50] E. Dudas, Y. Mambrini, and K. A. Olive, Phys. Rev. D 91, 075001 (2015).

[51] L. Heurtier, Y. Mambrini, and M. Pierre, Phys. Rev. D 99, 095014 (2019).

[52] J. M. Cline, C. Gross, and W. Xue, Phys. Rev. D 100, 015031 (2019); B. Chauhan and S. Mohanty, Phys. Rev. D 99, 095018 (2019); D. Hooper, S. Wegsman, C. Deaconu, and A. Vieregg, Phys. Rev. D 100, 043019 (2019); L. Heurtier, D. Kim, J. C. Park, and S. Shin, Phys. Rev. D 100, 055004 (2019); L. A. Anchordoqui and I. Antoniadis, Phys. Lett. B 790, 578 (2019); L. A. Anchordoqui, V. Barger, J. G. Learned, D. Marfatia, and T. J. Weiler, Lett. High Energy Phys. 1, 13 (2018); J. H. Collins, P. S. Bhupal Dev, and Y. Sui, Phys. Rev. D 99, 043009 (2019).

[53] M. Ackermann et al. (Fermi-LAT Collaboration), Phys. Rev. D 88, 082002 (2013); T. Cohen, K. Murase, N. L. Rodd, B. R. Safdi, and Y. Soreq, Phys. Rev. Lett. 119, 021102 (2017); M. G. Baring, T. Ghosh, F. S. Queiroz, and K. Sinha, Phys. Rev. D 93, 103009 (2016); M. Cirelli, E. Moulin, P. Panci, P. D. Serpico, and A. Viana, Phys. Rev. D 86, 083506 (2012).

[54] A. Ibarra, A. S. Lamperstorfer, and J. Silk, Phys. Rev. D 89, 063539 (2014).

[55] M. G. Aartsen et al. (IceCube Collaboration), Eur. Phys. J. C 78, 831 (2018); M. Kachelriess, O. E. Kalashev, and M. Y.
Kuznetsov, Phys. Rev. D 98, 083016 (2018); M. Y. Kuznetsov, JETP Lett. 105, 561 (2017).

[56] N. Palanque-Delabrouille, C. Yèche, N. Schöneberg, J. Lesgourgues, M. Walther, S. Chabanier, and E. Armengaud, J. Cosmol. Astropart. Phys. 04 (2020) 038.

[57] S. Vagnozzi, E. Giusarma, O. Mena, K. Freese, M. Gerbino, S. Ho, and M. Lattanzi, Phys. Rev. D 96, 123503 (2017); S. Roy Choudhury and S. Choubey, J. Cosmol. Astropart. Phys. 09 (2018) 017.

[58] V. Berezinsky and J. W. F. Valle, Phys. Lett. B 318, 360 (1993); M. Lattanzi and J. W. F. Valle, Phys. Rev. Lett. 99, 121301 (2007); F. Bazzocchi, M. Lattanzi, S. RiemerSorensen, and J. W. F. Valle, J. Cosmol. Astropart. Phys. 08 (2008) 013; M. Lattanzi, S. Riemer-Sorensen, M. Tortola, and J.W.F. Valle, Phys. Rev. D 88, 063528 (2013); M. Lattanzi, R. A. Lineros, and M. Taoso, New J. Phys. 16, 125012 (2014); J. Gehrlein and M. Pierre, J. High Energy Phys. 02 (2020) 068.

[59] I. Z. Rothstein, K. S. Babu, and D. Seckel, Nucl. Phys. B403, 725 (1993).

[60] A. Linde, Y. Mambrini, and K. A. Olive, Phys. Rev. D 85, 066005 (2012).

[61] S. Dimopoulos, SUSY98, Oxford; K. R. Dienes, E. Dudas, and T. Gherghetta, Nucl. Phys. B557, 25 (1999); N. ArkaniHamed, S. Dimopoulos, G. R. Dvali, and J. March-Russell, Phys. Rev. D 65, 024032 (2001).

[62] P. Minkowski, Phys. Lett. B 67, 421 (1977); T. Yanagida, Conf. Proc. C 7902131, 95 (1979); M. Gell-Mann, P. Ramond, and R. Slansky, Conf. Proc. C 790927, 315 (1979); S. L. Glashow, NATO Sci. Ser. B 59, 687 (1980); R. N. Mohapatra and G. Senjanovic, Phys. Rev. Lett. 44, 912 (1980); Phys. Rev. D 23, 165 (1981); J. Schechter and J. W. F. Valle, Phys. Rev. D 22, 2227 (1980); 25, 774 (1982).

[63] K. Ishiwata, O. Macias, S. Ando, and M. Arimoto, J. Cosmol. Astropart. Phys. 01 (2020) 003.

[64] M. Kachelriess, O. E. Kalashev, and M. Y. Kuznetsov, Phys. Rev. D 98, 083016 (2018).

[65] B. D. Fields, K. A. Olive, T. Yeh, and C. Young, J. Cosmol. Astropart. Phys. 03 (2020) 010.

[66] G. Steigman, K. A. Olive, and D. Schramm, Phys. Rev. Lett. 43, 239 (1979); K. A. Olive, D. N. Schramm, and G. Steigman, Nucl. Phys. B180, 497 (1981).

[67] J. H. Adams, L. A. Anchordoqui, J. A. Apple, M. E. Bertaina, M. J. Christl, F. Fenu, E. Kuznetsov, A. Neronov, A. V. Olinto, T. C. Paul, P. Reardon, I. Vovk, L. Wiencke, and R. M. Young, arXiv:1703.04513.

[68] K. V. Berghaus, M. D. Diamond, and D. E. Kaplan, J. High Energy Phys. 05 (2019) 145.

[69] A. Olinto et al. arXiv:1907.06217.

[70] J. F. Navarro, C. S. Frenk, and S. D. M. White, Astrophys. J. 462, 563 (1996).

[71] R. Catena and P. Ullio, J. Cosmol. Astropart. Phys. 08 (2010) 004.

[72] A. Connolly, R. S. Thorne, and D. Waters, Phys. Rev. D 83, 113009 (2011). 